\title{
LESÃO POR ABRASÃO APÓS CATETERISMO CARDÍACO: RELATO DE CASO
}

\author{
Abrasive injury after cardiac catheterism: case report \\ Lesión por abrasión después del cateterismo cardíaco: relato de caso \\ Eduardo Tavares Gomes ${ }^{1 *}$, Maria de Andrade Lima Pitta Marinho², Mayana Camila Barbosa Galvão ${ }^{3}$, \\ Daniella Patricia Candido Rego ${ }^{4}$, Jackeline Alcoforado Vieira ${ }^{5}$, Maria Luciene dos Santos ${ }^{6}$
}

RESUM0: Objetivo: Relatar um caso de lesão de pele por abrasão relacionada à retirada de curativo pós-cateterismo cardíaco via artéria femoral. Método: Relato de um caso registrado em um hospital universitário do Nordeste do Brasil, ocorrido em setembro de 2017. Resultados: A paciente foi submetida a um cateterismo cardíaco, que indicou cirurgia de revascularização miocárdica. No período pré-operatório, identificou-se a lesão de pele, que se estendia transversalmente no abdome, na região infraumbilical e na face interna da coxa direita, correspondendo à região em que foi realizado o curativo compressivo pós-cateterismo. Conclusão: O curativo compressivo pós-cateterismo deve ser realizado e a enfermagem deve dar atenção especial à sua retirada, em virtude do risco de lesão por abrasão, que pode acometer uma grande área, ocasionando dano direto ao paciente e aumentando o risco de infecção. Palavras-chave: Complicações pós-operatórias. Ferimentos e lesões. Cateterismo cardíaco. Cirurgia torácica. Cuidados de enfermagem.

ABSTRACT: Objective: To report a case of skin abrasion related to dressing removal after cardiac catheterization via the femoral artery. Method: Report of a case registered in a university hospital in Northeastern Brazil, occurred in September 2017. Results: The patient was submitted to cardiac catheterization, which indicated myocardial revascularization surgery. In the preoperative period, the skin lesion was identified, extending transversely in the abdomen, infraumbilical region and the inner side of the right thigh, corresponding to the region where the post-catheterization compressive dressing was performed. Conclusion: The post-catheterization compressive dressing should be performed and nursing should pay special attention to its removal, due to the risk of abrasion, which can affect a large area, causing direct damage to the patient and increasing the risk of infection.

Keywords: Postoperative complications. Wounds and injuries. Cardiac catheterization. Thoracic surgery. Nursing care.

RESUMEN: Objetivo: Informar un caso de lesión de la piel relacionada con la extracción del apósito después del cateterismo cardíaco a través de la arteria femoral. Método: El informe de un caso registrado en un hospital universitario en el noreste de Brasil que ocurrió en septiembre de 2017. Resultados: El paciente fue sometido a cateterismo cardíaco, lo que indicó cirugía de revascularización miocárdica. En el período preoperatorio, se identificó la lesión de la piel, que se extiende transversalmente en el abdomen, la región infraumbilical y el lado interno del muslo derecho, correspondiente a la región donde se realizó el apósito compresivo post-cateterismo. Conclusión: El apósito compresivo post-cateterización debe realizarse y la enfermería debe prestar especial atención a su extracción, debido al riesgo de abrasión, que puede afectar a un área grande, causando daño directo al paciente y aumentando el riesgo de infección.

Palabras clave: Complicaciones postoperatorias. Heridas y lesiones. Cateterismo cardíaco. Cirugía torácica. Atención de enfermería.

'Enfermeiro; mestre em Enfermagem; especialista em Enfermagem em Suporte Avançado de Vida e em Enfermagem em Cardiologia; enfermeiro assistencial do Centro Cirúrgico do Hospital das Clínicas da Universidade Federal de Pernambuco - Recife (PE), Brasil.

Enfermeira; mestranda em Gestão e Economia da Saúde, Universidade de Pernambuco; chefe da Unidade de Centro Cirúrgico do Hospital das Clínicas da Universidade Federal de Pernambuco - Recife (PE), Brasil. ${ }^{3}$ Enfermeira; mestre em Enfermagem; enfermeira assistencial do Centro Cirúrgico do Hospital das Clínicas da Universidade Federal de Pernambuco - Recife (PE), Brasil.

${ }^{4}$ Enfermeira assistencial do Centro Cirúrgico do Hospital das Clínicas da Universidade Federal de Pernambuco - Recife (PE), Brasil.

${ }^{5}$ Enfermeira; especialista em Enfermagem em Saúde da Criança; enfermeira assistencial do Centro Cirúrgico do Hospital das Clínicas da Universidade Federal de Pernambuco - Recife (PE), Brasil.

${ }^{6}$ Enfermeira; especialista em Enfermagem em Oncologia e em Enfermagem em Estomaterapia; enfermeira assistencial do Centro Cirúrgico do Hospital das Clínicas da Universidade Federal de Pernambuco Recife (PE), Brasil.

*Autor correspondente: edutgs@hotmail.com

Recebido: 07/11/2017 - Aprovado: 11/03/2018

DOl: 10.5327/Z1414-4425201800020009 


\section{INTRODUÇÃO}

O cateterismo cardíaco via artéria femoral, além dos riscos inerentes ao procedimento invasivo e ao uso do contraste, adiciona desconforto ao paciente que a ele se submete, devido à imobilidade requerida durante a presença do curativo compressivo ${ }^{1}$.

O cuidado de enfermagem prestado a pacientes que se submetem ao procedimento deve ser integral e atento às complicações ${ }^{2}$. Para evitar sangramentos, hematomas e equimoses no local de punção, um curativo compressivo é realizado de forma reforçada, com grande extensão, podendo chegar a ter efeito de garrote sobre a coxa. Após o cateterismo, os pacientes, na maioria dos serviços no país, mantêm a imobilidade e a restrição ao leito, enquanto permanecem com esse curativo por até seis horas, mesmo que já existam evidências de que esse tempo pode ser reduzido com segurança para até três horas ${ }^{3,4}$.

As complicações do procedimento já estão bem documentadas. No entanto, não se encontrou, na literatura consultada, referência à lesão por abrasão na retirada do curativo compressivo, possivelmente por ser um evento de rara ocorrência, além de ser um evento adverso não específico para o cateterismo ${ }^{3-6}$, fato que justifica a realização do presente estudo.

\section{OBJETIVO}

Relatar um caso de lesão de pele por abrasão relacionada à retirada de curativo pós-cateterismo cardíaco via artéria femoral.

\section{MÉTODO}

Trata-se de relato de um caso registrado em um hospital universitário no Nordeste do Brasil, ocorrido em setembro de 2017. Descreve-se um caso de lesão de pele por abrasão relacionada à retirada de curativo pós-cateterismo cardíaco via artéria femoral, como ocorreu o evento adverso, a relevância e as implicações desse no período perioperatório de cirurgia cardíaca.

A paciente foi avaliada em dois momentos: primeiramente, na véspera da cirurgia, 12 dias após o cateterismo diagnóstico, e no intraoperatório, quando realizou a cirurgia cardíaca.

Para o apontamento dos dados, foram consultados os registros no prontuário, além da avaliação pré e intraoperatória, por parte dos pesquisadores. No período pré-operatório, na véspera da cirurgia, houve uma visita de enfermagem realizada por uma enfermeira estomaterapeuta, que descreveu o estado da lesão e registrou o risco da paciente ser submetida à cirurgia cardíaca.
O relato do caso faz parte do projeto de investigação sobre as lesões de posicionamento cirúrgico e de pele no período perioperatório, aprovado pelo Comitê de Ética em Pesquisa (CEP) da instituição sede do estudo (CAAE: 66142117.0.0000.5208, Parecer: 2.045.355). Salienta-se que a paciente, foco do estudo, concordou com a realização do mesmo e com a publicação das imagens. Os autores se comprometem a utilizar as informações para realização deste estudo e a manter o anonimato do sujeito da pesquisa.

\section{RESULTADOS}

A paciente idosa, 67 anos, obesa (índice de massa corporal $\mathrm{IMC}=36,3 \mathrm{~kg} / \mathrm{m}^{2}$ ), hipertensa, diabética não insulinodependente, não tabagista, não etilista, referia dispneia paroxística noturna e edema de membros inferiores há cerca de 3 anos, sem investigação da causa.

Apresentou quadro de dor torácica aguda, sendo encaminhada à emergência cardiológica de referência, após ser atendida em uma unidade de pronto-atendimento. Durante o atendimento pré-hospitalar, permaneceu hemodinamicamente estável, com queixa de dor retroesternal em aperto, que perdurou por mais de 12 horas, acompanhada de dispneia, com saturação parcial de oxigênio - Sat $\mathrm{O}_{2}>94 \%$. Os eletrocardiogramas apresentavam novo bloqueio de ramo direito e alterações indicativas de infarto agudo do miocárdio (IAM), com elevação correspondente dos marcadores de necrose miocárdica. Foi realizada estratégia de compensação da paciente e, em seguida, deu-se encaminhamento ao cateterismo cardíaco.

O procedimento foi realizado sem caráter de urgência, via artéria femoral, com introdutor 6 French, incidências de rotina para avaliação das coronárias e ventriculografia. Não houve registro em prontuário nem da retirada do introdutor, nem do curativo realizado pela enfermagem após o procedimento.

No dia seguinte ao cateterismo, realizou-se um ecocardiograma, que confirmou a indicação da cirurgia de revascularização miocárdica $(\mathrm{RM})$. A cirurgia foi agendada para 10 dias após a confirmação cirúrgica, 12 dias após o cateterismo.

$\mathrm{Na}$ véspera da cirurgia, a paciente recebeu a visita de enfermagem pré-operatória de rotina, na qual os pacientes são avaliados, recebem instruções a respeito do perioperatório e podem tirar suas dúvidas. Na visita, a enfermeira do centro cirúrgico (CC) identificou as lesões e solicitou avaliação da enfermeira estomaterapeuta.

A lesão denotava características de abrasão da pele na área correspondente ao curativo compressivo, comumente realizado na região após procedimentos hemodinâmicos. Estendia-se infraumbilical 
e transversalmente (Figura 1), e se projetava pelas faces anterior e interna da coxa direita (Figura 2), sem sinais de infecção, com bordas em retração e sinais de reepitelização. A enfermeira estomaterapeuta registrou em prontuário, comunicou à enfermeira do setor e entrou em contato com o médico responsável pela cirurgia cardíaca, que confirmou estar ciente e que a paciente deveria ser submetida à cirurgia mesmo assim, em virtude do risco de se protelar mais esse segundo procedimento.

No dia da cirurgia, a paciente chegou ao CC após preparo na enfermaria com banho pré-operatório, tricotomia, incluindo membros inferiores, e acesso venoso periférico. Ao entrar em sala operatória (SO), a paciente foi colocada na mesa adequada, respondeu ao checklist de cirurgia segura, realizado pelo enfermeiro, e recebeu a indução anestésica. Em seguida, procedeuse à intubação orotraqueal, punção venosa central e de artéria radial esquerda para monitoração invasiva da pressão arterial.

Após os procedimentos realizados pela equipe da anestesia, incluindo a antibioticoterapia profilática, o enfermeiro despiu a paciente para realizar a sondagem vesical e o preparo da pele, deparando-se com a extensa lesão. A equipe discutiu e procedeu-se em virtude da relação risco-benefício ponderada pelo cirurgião. No instrumento para registro da

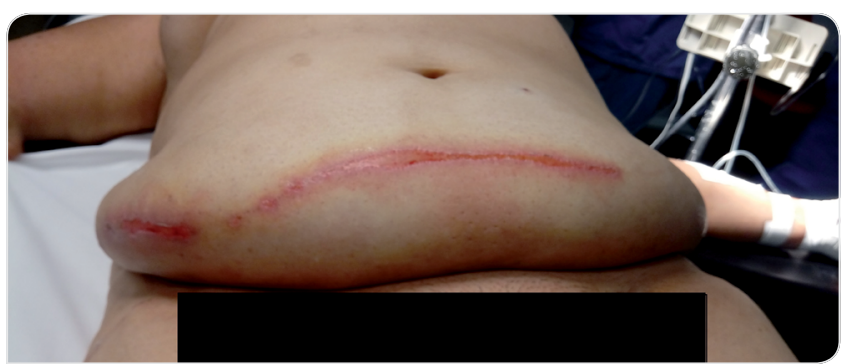

Figura 1. Lesão por abrasão na região abdominal infraumbilical, após retirada de curativo compressivo realizado com esparadrapo pós-cateterismo cardíaco.

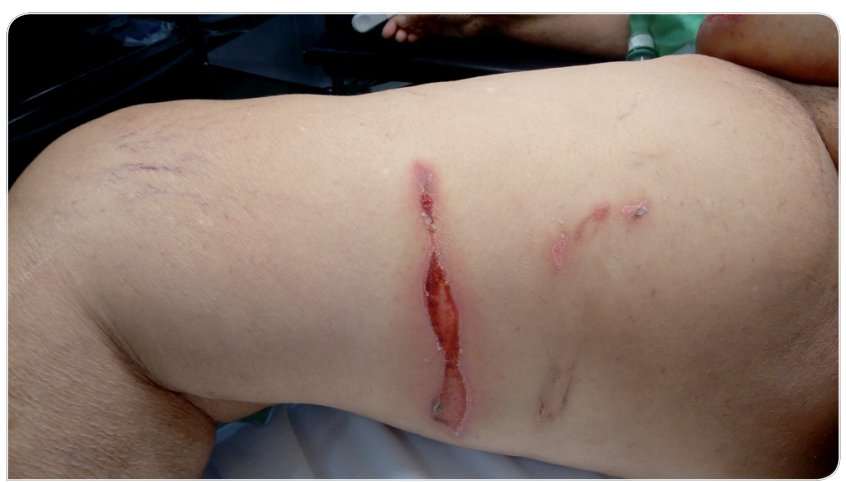

Figura 2. Lesão por abrasão na face interna da coxa direita, após retirada de curativo compressivo realizado com esparadrapo pós-cateterismo cardíaco.
Sistematização da Assistência de Enfermagem Perioperatória (SAEP), o enfermeiro verificou os registros da visita pré-operatória e registrou o estado da ferida na admissão em sala.

Durante o posicionamento para cirurgia cardíaca, os pacientes são, no serviço, rotineiramente envolvidos numa faixa de esparadrapo, fixada nas extremidades à mesa cirúrgica. Em outras cirurgias, as faixas com velcro e presilhas são utilizadas; contudo, como na cirurgia cardíaca é realizada a degermação de todo o tronco, a faixa de esparadrapo é utilizada de forma a permitir que a solução seja aplicada continuamente; não há como utilizá-la com a faixa com velcro.

Para o preparo da pele, foi empregada a solução de clorexidina degermante, seguida por solução alcoólica. Houve o cuidado para que esta não entrasse em contato com a área de lesão, mas a mesma recebeu a solução degermante, uma vez que não havia solução aquosa disponível. Entre a primeira degermação, realizada pelo enfermeiro, e uma segunda, feita pelo instrumentador, a região do abdome recebeu uma camada de compressa transversalmente, sobre a qual se passou a faixa de esparadrapo para segurança no posicionamento cirúrgico, garantindo que não houvesse novo contato entre a pele e o esparadrapo, de forma a evitar piora ou ocorrência de novas abrasões.

O período intraoperatório durou aproximadamente quatro horas, com extubação em SO e transferência da paciente com monitoração e sem drogas vasoativas para a unidade de recuperação da cirurgia cardíaca. Na retirada da faixa de esparadrapo, observou-se que não houve nova lesão. Ao final da cirurgia, as bordas da lesão e a pele peri-incisional estavam hiperemiadas, provavelmente pelo tempo em que se mantiveram com a solução de clorexidina.

Após a cirurgia, foi realizada a notificação do evento adverso. A paciente não teve infecção de sítio cirúrgico (ISC) e nem outras complicações infecciosas no período de 11 dias de internação, até que receber alta hospitalar.

\section{DISCUSSÃO}

A paciente apresentava fatores de risco para lesões de pele e posicionamento, aos quais deveria ser dada maior atenção, como a diabetes, a idade e a obesidade ${ }^{7}$.Tais fatores já devem servir de alerta para a necessidade de estratégias de prevenção de lesão de pele e lesões por posicionamento, que incluem inspeção criteriosa inicial do estado da derme e dos riscos.

Uma estratégia de que alguns enfermeiros lançam mão para proteger a pele, no que é possível, é realizar uma primeira cobertura do curativo compressivo pós-cateterismo ou angioplastia com 
fita microporosa, para só depois cobrir com esparadrapo, evitando o contato epidérmico e facilitando a remoção. Destaca-se que não foram encontrados na literatura estudos que validem esse recurso.

Não é possível afirmar que o serviço de hemodinâmica falhou no checkout, ou seja, na conferência do paciente na saída da sala de procedimentos, visto que a enferma teve alta do serviço para a emergência, quando foi realizada a retirada do curativo ${ }^{8,9}$. Relatos de abrasão depois desse procedimento também não foram encontrados na literatura.

Um estudo de revisão integrativa verificou a prevalência de 27 diagnósticos de enfermagem utilizados em serviços de hemodinâmica; contudo, na revisão não foram encontrados os diagnósticos de "risco de integridade da pele prejudicada" e nem "risco de integridade tissular prejudicada”, ambos aplicáveis em complicações, sendo o primeiro útil à descrição da lesão de pele por abrasão e, o segundo, de complicações, como hematomas ${ }^{10}$. Outro estudo, apesar de encontrar o uso do diagnóstico de enfermagem "risco de integridade da pele prejudicada” para pacientes pós-cateterismo, aponta que o exame foi citado por poder se utilizar dele para qualquer invasão da derme, sem considerar a ameaça de abrasão ${ }^{11}$.

A experiência de outro serviço foi relatada em uma publicação que descreve a aplicabilidade de um checklist elaborado para acompanhar o procedimento de cateterismo cardíaco diagnóstico e terapêutico como estratégia para o gerenciamento do cuidado de enfermagem, evidenciando melhora da qualidade da assistência e sugerindo que essa ferramenta pode ser utilizada até o momento da retirada do curativo, quando se avalia a possiblidade de complicações no local de punção que dificilmente seriam visualizadas antes ${ }^{12}$.

No CC, o timeout, ou a checagem da segurança do paciente imediatamente antes da incisão da pele, é um desafio ainda para a enfermagem, que deveria incluir a avaliação integral do paciente, com registro de lesões de pele prévias e revisão das estratégias de prevenção de lesões novas e do posicionamento cirúrgico $^{7,8,13}$. Demandas como montagem de sala, atraso nas cirurgias e colaboração da equipe dificultam essa etapa ${ }^{8,14}$. No caso apresentado, o enfermeiro do CC identificou a lesão apenas após a intubação e a realização de procedimentos invasivos.

Em uma revisão integrativa com o objetivo de identificar os cuidados de enfermagem no posicionamento cirúrgico, relatando as complicações, não foi descrito como cuidado evitar o uso de esparadrapo, apesar do mesmo ainda ser utilizado em algumas cirurgias, em particular para garantir a segurança do paciente quando há movimentação da mesa para inclinação lateral, elevação da cabeça ou membros inferiores durante a cirurgia, ou quando o paciente já é acomodado nessas posições ${ }^{7}$. No CC onde ocorreu o caso, tem-se disponíveis faixas com velcro e presilhas para esse fim; contudo, ainda há equipes que preferem usar esparadrapo.

Ao final da cirurgia, a ocorrência foi notificada no sistema informatizado de vigilância hospitalar para eventos adversos. Esse procedimento deve ser incorporado à rotina dos profissionais de saúde, visto que, apesar de altos índices de eventos adversos serem registrados em alguns estudos, sabe-se que esses resultados podem ser subestimados quando não há cultura organizacional que subsidie a adesão aos registros ${ }^{15}$.

\section{CONSIDERAÇÕES FINAIS}

O curativo compressivo pós-cateterismo deve ser realizado e a enfermagem deve dar atenção especial à sua retirada, em virtude do risco de lesão por abrasão que pode acometer uma grande área, ocasionando dano direto e aumentando o risco de infecção, como ocorreu com a paciente referida neste estudo.

Sugere-se o estabelecimento de protocolos de prevenção de lesões de pele, tanto para os serviços de hemodinâmica quanto para os CC. Por fim, reforça-se a importância da notificação do evento adverso para análise das falhas, delineamento de intervenções e reflexão constante sobre protocolos e rotinas institucionais.

\section{REFERÊNCIAS}

1. Dal Piva C, Vaz E, Moraes MA, Goldmeyer S, Linch GFC, Souza EN. Desconfortos relatados pelos pacientes após cateterismo cardíaco pelas vias femoral ou radial. Rev Bras Cardiol Invasiva. 2014;22(1):3640. DOI: 10.1590/0104-1843000000008

2. Aguiar BF, Rinaldi ECA, Cintho LMM, Martins CLS, Zimmerman MH. Importância dos cuidados de enfermagem no cateterismo cardíaco. Cienc Cuid Saude. 2016;15(3):460-5. http://dx.doi.org/10.4025/ cienccuidsaude.v15i3.24894
3. Matte R, Hilário TS, Reich R, Aliti GB, Rabelo-Silva ER. Reducing bed rest time from five to three hours does not increase complications after cardiac catheterization: the Three Cath Trial. Rev Latinoam Enferm. 2016;24:e2797. http://dx.doi.org/10.1590/1518-8345.0725.2796

4. Rocha VS, Aliti R, Moraes MA, Rabelo ER. Three-hour rest period after cardiac catheterization with a $6 \mathrm{~F}$ sheath does not increase complications: a randomized clinical trial. Rev Bras Cardiol Invasiva. 2009;17(4):5127. http://dx.doi.org/10.1590/S2179-83972009000400015 
5. Barbosa MH, Moreira TM, Tavares JL, Andrade EV, Bitencourt MN, Freitas KBC, et al. Complicaciones en pacientes sometidos a angioplastia coronaria transluminal percutánea. Enferm Glob. 2013;12(31):14-33.

6. Andrade PB, Andrade MVA, Barbosa RA, Labrunie A, Hernandes ME, Marino RL, et al. Femoral versus radial access in primary angioplasty: analysis of the accept registry. Arq Bras Cardiol. 2014;102(6):56670. https://dx.doi.org/10.5935\%2Fabc.20140063

7. Miranda $A B$, Fogaça AR, Rizzetto M, Lopes LCC. Surgical positioning: nursing care in the transoperative period. Rev SOBECC. 2016;21(1):528. DOI: $10.5327 / Z 1414-4425201600010008$

8. Martins GS, Carvalho R. Realização do timeout pela equipe cirúrgica: facilidades e dificuldades. Rev SOBECC. 2014;19(1):18-25.

9. Giannattasio M, Taniguchi F. Avaliação da segurança do paciente em cirurgia cardíaca de um hospital público. Rev SOBECC. 2016;21(3):12531. https://doi.org/10.5327/Z1414-4425201600030002

10. Taets GGC. Cuidados de enfermagem e diagnósticos para pacientes submetido à angioplastia coronária transluminal percutânea. Rev Recien. 2016;6(16):3-10.
11. Aquino EM, Roehrs H, Méier MJ. Nursing diagnosis in patients undergoing a cardiac catheterization in a unit of cardiology. Rev Enferm UFPE. 2014;8(11):3929-37. https://doi. org/10.5205/1981-8963-v8i1 la13617p3929-3937-2014

12. Sousa SM, Bernardino E, Bueno RRL, Tironi NM, Mercês NNA, Aued GK. Checklist for monitoring of heart catheterization: a strategy for nursing management. Rev Enferm UFPE. 2015;9(12):1063-8. https:// doi.org/10.5205/1981-8963-v9i12a10808p1063-1068-2015

13. Carneiro GA, Leite RCBO. Skin lesions in the intraoperative period of cardiac surgery: incidence and characterization. Rev Esc Enferm USP. 2011;45(3):611-6. http://dx.doi.org/10.1590/ S0080-62342011000300009

14. Souza R, Araújo M, Veríssimo R, Comassetto I, Ferreira F, Bernardo T. Aplicabilidade do checklist de cirurgia segura em centros cirúrgicos hospitalares. Rev SOBECC. 2016;21(4):192-7. https://doi.org/10.5327/ Z1414-4425201600040003

15. Bohomol E, Tartali JA. Adverse effects in surgical patients: knowledge of the nursing professionals. Acta Paul Enferm. 2013;26(4):376-81. http://dx.doi.org/10.1590/S0103-21002013000400012 